\title{
Terahertz quantum Cascade Lasers Frequency combs: Wide bandwidth operation and dual-comb on a chip
}

\author{
G. Scalari ${ }^{1}$, M. Rösch ${ }^{1}$, M. Beck ${ }^{1}$, D. Bachmann ${ }^{2}$, K. Unterrainer ${ }^{2}$, J. Faist $^{1}$ \\ ${ }^{1}$ ETH Zurich, Institute of Quantum Electronics, Auguste-Piccard-Hof 1, CH-8093 Zurich, Switzerland \\ ${ }^{2}$ TU Wien, Photonics Institute and Center for Micro- and Nanostructures, Gußhausstraße 27-29, 1040 Vienna, Austria
}

Terahertz (THz) quantum-cascade lasers (QCLs) constitute a very promising candidate for compact, wide bandwidth, integrated frequency combs [1,4-7]. QCLs based on heterogeneous cores display the widest spectral coverage reaching more than one octave [2]. The possibility to engineer the gain profile turns out to be fundamental also with respect to dispersion compensation in order to extended the comb spectral bandwidth [2,7]. In the effort of extending the comb operation to a full-octave to implement the laser self-referencing [3], we present here a new here a new THz QCL active region that allows the generation of a frequency comb with a spectral bandwidth exceeding of $1 \mathrm{THz}$ centered at $3.1 \mathrm{THz}$. It fully exploits the capability of QCLs to integrate different active region designs within one laser cavity. The used building block is the three-active region design reported in Ref. [2], where a design at $3.4 \mathrm{THz}$ has been added to increase the bandwidth towards higher frequencies. The four designs have central frequencies of 2.3, 2.6, 2.9, and $3.4 \mathrm{THz}$, the number of periods per design has also been rearranged in order to provide a flat gain resulting in a similar threshold for all the active regions and more dynamic range. Additionally, the doping level has been increased. Laser performance results in a largely extended dynamic range with respect to the original three-stack structure. Peak powers above $8 \mathrm{~mW}$ are recorded at $30 \mathrm{~K}$ and the lasing spectrum spans over $1.94 \mathrm{THz}$ from $1.88 \mathrm{THz}$ to $3.82 \mathrm{THz}$ covering more than a full octave in frequency (Fig.1(a,b)). Dry-etched lasers with side-absorbers for lateral mode suppression similar as in [5] were fabricated for continuous wave (CW) operation and comb operation is probed through the beatnote analysis. Fig. 1(c,d) shows the beatnote as a function of the injected current with a maximum comb span of $1.1 \mathrm{THz}$ which is the broadest demonstrated so far. Further proof of comb regime comes from the simultaneous measurements of two laser ridges on the same chip that show multiheterodyne spectra working in a dual-comb configuration [6].
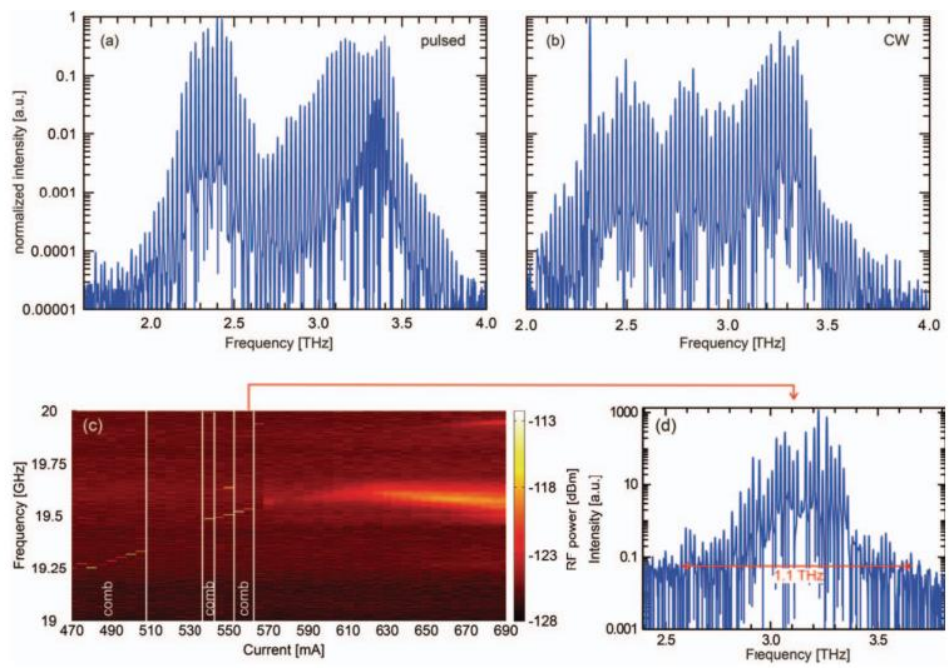

Fig. 1 (a): Optical spectrum of a $1.5 \mathrm{~mm}$ x $150 \mu \mathrm{m}$ wet-etched laser in pulsed (20\% duty cycle) at 20 Kelvin. (b) Optical spectrum of a $1.8 \mathrm{~mm}$ x $60 \mu \mathrm{m}$ dry-etched laser in CW operation at 21 Kelvin. (c) Intermode beatnote of a $2 \mathrm{~mm}$ x $60 \mu \mathrm{m}$ dry-etched laser as a function of the driving current. (T=20 K). The beatnote signal is extracted from the bias line with a bias-tee (RBW): $10 \mathrm{kHz},(\mathrm{VBW})$ : $100 \mathrm{kHz}, \mathrm{sweep}$ time (SWT): 10 sec.). (d) Optical spectrum for the same laser at $562 \mathrm{~mA}$ at 16 Kelvin. For this current the laser is in a FC regime providing a bandwidth of 1.1 THz.

\section{References}

[1] D. Burghoff et al., Nature Photonics 8, 462-467 (2014)

[2] M. Rösch, G. Scalari, M. Beck, and J. Faist, Nature Photonics 9, 42-47 (2015)

[3] T. Udem, R. Holzwarth, T. W. Hänsch, Nature 416, 233 (2002).

[4] J. Faist, G. Villares, G. Scalari, M. Rösch, C. Bonzon, A. Hugi, and M. Beck, Nanophotonics 5, 272 (2016).

[5] D. Bachmann, M. Rösch et al., Optica 3, 1087-1094 (2016)

[6] M. Rösch, G. Scalari, G. Villares, L. Bosco, M. Beck, J. Faist, Appl. Phys. Lett. 108, 171104 (2016)

[7] D. Bachmann, M. Rösch, G. Scalari et al., Appl. Phys. Lett. , 109, 221107 (2016) 
(C) (C) 2017 IEEE. Personal use of this material is permitted. Permission from IEEE must be obtained for all other uses, in any current or future media, including reprinting/republishing this material for advertising or promotional purposes, creating new collective works, for resale or redistribution to servers or lists, or reuse of any copyrighted component of this work in other works.

This is the accepted version of G. Scalari, "Terahertz quantum Cascade Lasers Frequency combs: Wide bandwidth operation and dual-comb on a chip," 2017 Conference on Lasers and Electro-Optics Europe \& European Quantum Electronics Conference (CLEO/Europe-EQEC), Munich, 2017, doi : 10.1109/CLEOE-EQEC. 2017.8086427 